\title{
Extended inflation with an exponential potential
}

\author{
Mikel Susperregi \\ Astronomy Unit, School of Mathematical Sciences, Queen Mary \& Westfield College, University of London, \\ London E1 4NS, United Kingdom \\ Anupam Mazumdar \\ Astronomy Centre, University of Sussex, Falmer, Brighton BN1 9QJ, United Kingdom
}

(Received 5 May 1998; published 22 September 1998)

\begin{abstract}
In this paper we investigate extended inflation with an exponential potential $V(\sigma)=V_{0} e^{-\kappa \sigma}$, which provides a simple cosmological scenario where the distribution of the constants of nature is mostly determined by $\kappa$. In particular, we show that this theory predicts a uniform distribution for the Planck mass at the end of inflation, for the entire ensemble of universes that undergo stochastic inflation. Eternal inflation takes place in this scenario for a broad family of initial conditions, all of which lead up to the same value of the Planck mass at the end of inflation. The predicted value of the Planck mass is consistent with the observed value within a comfortable range of values of the parameters involved.
\end{abstract}

[S0556-2821(98)03620-0]

PACS number(s): 98.80.Cq

\section{INTRODUCTION}

The idea of a varying $G$ based on anthropic arguments, or what would eventually become concisely enunciated anthropic arguments, dates back to Dirac [1] and later Sciama [2]. Their ideas paved the way for Brans and Dicke to formulate a very interesting theory of gravity [3] that is described by the metric tensor $g_{\mu \nu}$ and a scalar field $\Phi$. In their theory the Planck mass over an ensemble of universes is given by $M_{\mathrm{p}}^{2}(\Phi)=16 \pi \Phi$, and the Brans-Dicke (BD) coupling $\omega$ determines the validity of the principle of equivalence in gravitation. In the limit $\omega \rightarrow \infty$ and a suitably large $\Phi$, BD gravity is equivalent to general relativity (GR). The theory predicts very small variations of $G$ within the horizon, but the value of $\omega$ is likely to leave its imprint on the cosmic microwave background (CMB) and in the different stages of the evolution of the Friedmann-Robertson-Walker (FRW) universe, such as the matter-radiation transition [4]. For larger scales, our implicit expectation is that although BD gravity is almost indistinguishable from GR in our observable universe, gravity may behave in a very different way in regions that are very distant from ours.

Inflation has been on its own a very important tool in describing the early universe, and combined with BD gravity it enables us to envisage a very interesting quantum cosmological scenario where we can address the question of what are the typical values of the constants of nature, either by means of anthropic arguments or by choosing suitable inflationary potentials. In chaotic inflation (for a review see e.g. [5]) the scalar field that governs inflation starts out from large values and its classical "slow-roll" motion along the slope of the potential towards the vacuum state is combined with quantum fluctuations. The fluctuations are stochastic [6] and they are responsible for continuously creating regions where inflation prevails, thus perpetuating the process indefinitely. The BD field influences the course of inflation, and the dynamical interplay of both scalar fields determines therefore the different stages of inflation, such as the begin- ning and end of inflation, the epoch when the classical drift and quantum fluctuations become comparable, etc. In extended inflation both fields evolve stochastically and the resulting distribution is to a large extent dependent on the potential. It can be argued that the assumption of starting out with large scalar fields in chaotic inflation is somewhat arbitrary. It can be shown however, that even though the physics may favor initial configurations where the fields are small (e.g. in the case of instanton solutions [7]), a Gaussian distribution of initial conditions around $\sigma \approx 0$ over a sufficiently broad ensemble of regions will result in an inflationary universe dominated by the largest values of $\sigma$ on the tails of this distribution, however strongly suppressed.

Extended inflation has been investigated by a number of authors [8-10], mostly in the context of first-order phase transitions of old inflation, where the so-called big-bubble problem arises as first pointed out by Weinberg [11]. Later papers have focused on chaotic inflation, by computing the distributions of the fields, spectrum of density fluctuations, etc. $[12,13]$, where transitions are second-order and there are no bubbles or discontinuous interfaces except for those created by quantum fluctuations.

As it has been pointed out in [14-16], the structure of the universe and values of the constants of nature, as derived from the distributions of the fields, depend on a very specific and crucial feature of the inflation potential. In the plane $(\sigma, \Phi)$ it is easy to compute for each potential the end-ofinflation (EoI) and beginning-of-inflation (BoI) curves. The region of the plane enclosed between these two curves determines the range of values of the fields for which inflation will take place. The classical trajectories span from BoI and cross EoI, but the quantum fluctuations allow jumps of the fields between neighboring classical trajectories, and therefore the allowed states undergoing inflation quickly spread over the region enclosed between the curves BoI and EoI. The main characteristic of a potential is whether the area enclosed between BoI and EoI is finite or infinite. In [16], these are named class I and class II respectively. A class II 
potential yields to a universe where the spectrum of perturbations is arbitrarily small and the likelihood of a finite value of the Planck mass is negligible. In these theories, the values of the fields grow without limit, and any reasonable physical prediction becomes impossible without resorting to very stringent anthropic arguments. On the other hand, in the case of class I potentials BoI and EoI cross, and it is easy to show that the predominant values of the fields are those corresponding to the configuration exactly located at the crossing point. In this case, it is possible to predict values of the constants of nature that are perfectly consistent with the observed values, and that comes out naturally from the physics, without an exaggerated use of anthropic arguments. In this paper we show that the exponential potential is a class I potential.

In Sec. 2 we discuss the classical trajectories of the fields in the slow-roll approximation and the form of the BoI and EoI curves, which are the delimiters of inflation on the $(\sigma, \Phi)$ plane. In Sec. 3 we compute the probability distributions $P(\sigma, \Phi)$, volume ratios of homogeneous hypersurfaces, and finally in Sec. 4 we derive the corresponding spectrum of density fluctuations discussing typical values of the parameters and consistency with the astrophysical constraints.

\section{CLASSICAL TRAJECTORIES}

The extended inflation action is given by [8]

$$
S=\int d^{4} x \sqrt{-g}\left[\Phi R-\frac{\omega}{\Phi}(\partial \Phi)^{2}-\frac{1}{2}(\partial \sigma)^{2}-V(\sigma)\right],
$$

where $R$ is the curvature scalar and the potential is $V(\sigma)$ $=V_{0} e^{-\kappa \sigma}$. The coupling $\omega$ plays a similar role as that of the coupling functions $B_{i}(\Psi)$ of the dilaton field $\Psi$ in string theory. Based on this analogy, several authors have investigated the so-called hyperextended inflation models $[9,10,14]$, where $\omega$ becomes dependent on the BD field. In this paper however, we will merely examine the $\omega=$ const model. The BoI boundary is given by $V(\sigma)=M_{\mathrm{p}}^{4}(\Phi)$ or equivalently

$$
\Phi=\frac{V_{0}^{1 / 2}}{16 \pi} e^{-\kappa \sigma / 2}
$$

The BoI is the quantum limit where the metric fluctuations become significant and the inflation field cannot take the values for which the potential is above this boundary. The EoI boundary is on the other hand

$$
\frac{1}{2} \dot{\sigma}^{2}+\omega \frac{\dot{\Phi}^{2}}{\Phi} \approx V(\sigma)
$$

The equations of motion in a flat FRW background are

$$
\begin{gathered}
\left(D^{2}+\frac{1}{2 \omega} R\right) \Phi=0, \\
D^{2} \sigma=-V^{\prime}(\sigma),
\end{gathered}
$$

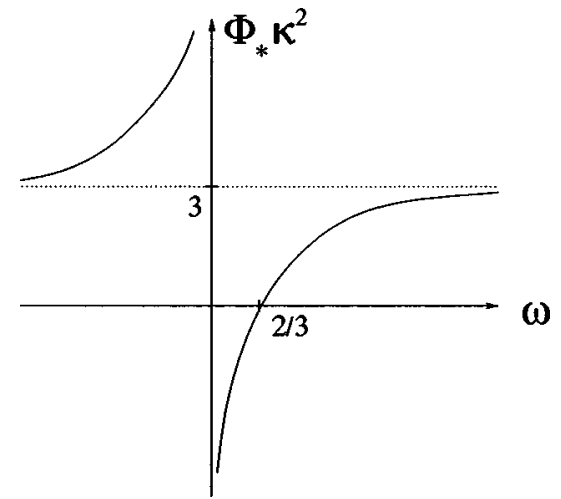

FIG. 1. BD field at EoI, $\Phi_{*}$, vs $\omega$ for an arbitrary value of $\kappa$. $\Phi_{*}$ is given in units of $\kappa^{-2}$. Inflation takes place in the range $\omega$ $<0$ or $\omega \geqslant 2 / 3$.

$$
H^{2}+H \frac{\dot{\Phi}}{\Phi}=\frac{\omega}{6}\left(\frac{\dot{\Phi}}{\Phi}\right)^{2}+\frac{1}{6 \Phi}\left[\frac{1}{2} \dot{\sigma}^{2}+V(\sigma)\right]
$$

and the differential operator $D$ is defined

$$
D^{2} \equiv \partial_{t}^{2}+3 H \partial_{t} .
$$

In the slow-roll approximation, $\ddot{\Phi} \ll H \dot{\Phi} \ll H^{2} \Phi$ and $\dot{\sigma}^{2}$ $+2 \omega \dot{\Phi}^{2} / \Phi \ll 2 V(\sigma)$, Eqs. (4)-(6) read

$$
\begin{aligned}
\frac{\dot{\Phi}}{\Phi} & =2 \frac{H}{\omega}, \\
\dot{\sigma} & =-\frac{1}{3 H} V^{\prime}(\sigma), \\
H^{2} & =\frac{1}{6 \Phi} V,
\end{aligned}
$$

and the curvature scalar is given by $R=-12 H^{2}$. The slowroll equations (8) enable us to rewrite Eq. (3):

$$
\Phi_{*}=\left(3-\frac{2}{\omega}\right) \frac{1}{\kappa^{2}}
$$

where the $*$ subindex denotes the value at the end of inflation. Hence, $\Phi_{*}$ is independent of $\sigma$ and, for reasonably large $\omega$, it is solely determined by the slope of the potential. The condition $\Phi>0$ also imposes the constraint $\omega$, as can be seen in Fig. 1, such that the range $0<\omega<2 / 3$ is excluded to prevent imaginary values of the Planck mass. The classical trajectories of the fields are given by the following conservation law [14]:

$$
\frac{d}{d t}\left[\omega \Phi+\int d \sigma \frac{V(\sigma)}{V^{\prime}(\sigma)}\right]=0
$$

which in the case of the exponential potential yields

$$
\Phi=\frac{\sigma}{\kappa \omega}+\left(\Phi_{0}-\frac{\sigma_{0}}{\kappa \omega}\right)
$$




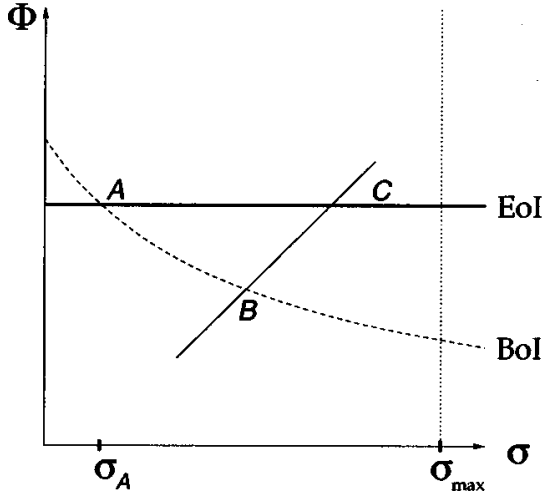

FIG. 2. Predicted BoI and EoI curves, dashed and thick solid curves respectively. Classical trajectories are straight lines parallel to $B C$. At the intersection point $A$ the onset and end of inflation coincide. $\sigma_{\max }$ determines the scale of validity of the slow-roll approximation. Inflation takes place within the region enclosed by BoI, EoI and $\sigma \approx \sigma_{\max }$.

In Fig. 2 we have put together the BoI and EoI curves and the classical trajectories of the fields on the $(\sigma, \Phi)$ plane, i.e. Eqs. (2), (11) and (13) respectively. It can be seen in the figure that inflation takes place in the region enclosed by BoI and EoI to the right of the intersection point $A$. The trajectories given by Eq. (13) are straight lines parallel to the segment $B C$, and the fields drift along these curves in the direction $B \rightarrow C$ during the course of inflation. The region enclosed by BoI and EoI to the left of $A$ does not undergo inflation, because the orientation of the classical trajectories is such that the fields would move from EoI towards BoI, which is not an acceptable solution. In addition to the classical trajectories quantum diffusion is responsible for the jumps of the fields between neighboring classical trajectories. It can be seen that, unlike with power-law potentials, for which $\sigma$ decreases as $\Phi$ increases during the course of inflation, in the case of the exponential potential both fields increase during the slow roll.

The EoI boundary (11) gives a definite and unique prediction for $\Phi_{*}$, and also it implies that if $\Phi_{0}>\Phi_{*}$ inflation will not occur. In the case of $0<\Phi_{0} \lesssim \Phi_{*}$, inflation takes place for values of the inflation

$$
\sigma_{0} \gtrsim-\frac{2}{\kappa} \log \left(\frac{16 \pi \Phi_{0}}{V_{0}^{1 / 2}}\right) .
$$

Naturally if $\Phi_{0}=\Phi_{*}$, then the right-hand side (RHS) of Eq. (14) is $\sigma_{A}$, the value of the field at the intersection point $A$ of BoI and EoI in Fig. 2.

It must be noted that the slow-roll approximation does not hold in the case of the exponential potential for arbitrarily large values of $\sigma$. For a given value of $\kappa$ it is straightforward to compute $\sigma_{\max }$ for which the potential and kinetic energy of the fields are comparable and thus the slow-roll conditions break down. It is easy to show from Eq. (3) that this scale is

$$
\sigma_{\max } \approx\left(\frac{3 \omega-2}{\kappa}\right) \text {. }
$$

Therefore it follows that the EoI boundary does not span from $\sigma_{A}$ to infinity, but inflation will occur within a finite region $\sigma_{A} \lesssim \sigma \lesssim \sigma_{\max }$.

\section{LIKELIHOOD RATIOS}

In the framework of extended inflation we can predict the distribution of $G$ in an ensemble of universes, depending on the scalar potential (see e.g. $[9,10,12]$ ). If the likeliest values predicted differ to a large extent from those observed in our region of the universe, then it becomes necessary to invoke the anthropic principle to justify the theory. On the other hand, a more optimal situation is achieved if the potential is able to yield maxima in the distribution of the fields that are compatible with the observed data. We have shown in the previous section that in the case of an exponential potential the outcome at the end of inflation is simple, and regardless of the initial conditions, a period of inflation leads to a uniform distribution of $\Phi$. As a result, regions that are still inflating will have values of the BD field in the range $0<\Phi$ $<\Phi_{*}$, those regions where inflation has ended have invariably $\Phi=\Phi_{*}$. There will be other regions whose initial conditions do not yield inflation (i.e. they are not located in the region enclosed by BoI and EoI in Fig. 2). The dominant contribution to the total volume will be given by the regions that are still inflating or have completed inflation in the recent past.

It is apparent from Fig. 2 that whereas the magnitude of $\Phi$ is bounded during the course of inflation, that of the inflation scalar is not. We can thus expect that quantum fluctuations may prolong the course of inflation by taking $\sigma$ to arbitrarily large values. In this section we compute the volume ratios of homogeneous hypersurfaces $(\sigma, \Phi)=$ const with respect to the total volume occupied by thermalized regions, using the results of [14]. As inflation is eternal, the volumes of the hypersurfaces are divergent, though the volume ratios are not. These ratios give a good measure of the relative likelihood of an arbitrary configuration $(\sigma, \Phi)$ with respect to another.

The comoving probability $P_{c}(\sigma, \Phi, t)$ is governed by the conservation equation [6]

$$
\partial_{t} P_{c}=-\partial_{\sigma} J_{\sigma}-\left(\frac{\Phi}{2 \omega}\right)^{1 / 2} \partial_{\Phi} J_{\Phi},
$$

where the probability current $\vec{J} \equiv\left(J_{\sigma}, J_{\Phi}\right)$ is given by the slow-roll solutions in the regime where quantum diffusion is neglected:

$$
\begin{gathered}
J_{\sigma} \approx-\frac{M_{\mathrm{p}}^{2}(\Phi)}{4 \pi} H^{-1} \partial_{\sigma} H P_{c}, \\
J_{\Phi} \approx-\frac{M_{\mathrm{p}}^{2}(\Phi)}{2 \pi}\left(\frac{\Phi}{2 \omega}\right)^{1 / 2} H^{-1} \partial_{\Phi} H P_{c},
\end{gathered}
$$

where $t$ corresponds to cosmic time. In order to solve Eq. (16), it is customary to adopt the eigenvalue expansion [10] 


$$
P_{c}(\sigma, \Phi, t)=\sum_{n=1}^{\infty} \psi_{n}(\sigma, \Phi) e^{-\gamma_{n} t}
$$

where $\gamma_{1}<\gamma_{2}<\gamma_{3}<\ldots$, and thus the asymptotic limit $t$ $\rightarrow \infty$ yields $P_{c} \sim \psi_{1} e^{-\gamma_{1} t}$. Substituting Eqs. (17),(18) in (16), we get [14]

$$
P_{c}(\sigma, \Phi, t) \approx C_{0} \Phi^{1 / 2} \exp \left(8 \pi \omega \gamma_{1} \Phi-\gamma_{1} t\right),
$$

where $C_{0}$ is a normalization constant and $\gamma_{1}$ is estimated numerically from the resulting eigenvalue equation for different values of $V_{0}$ and $\kappa$. For $V_{0} \sim 1$ and $0.1 M_{\mathrm{p}} \lesssim \kappa^{-1}$ $\lesssim 10 M_{\mathrm{p}}$, we have $2.4 \lesssim \gamma_{1} \lesssim 2.8$.

The volume $\mathcal{V}_{*}$ of thermalized regions over the entire spacetime is determined by the two-dimensional probability flux of the fields across the EoI boundary (11). The line element along EoI is $d \sigma$ and the differential flux is $d \sigma(\vec{J} \cdot \hat{n})$, where $\hat{n}$ is a normal vector to EoI. Hence

$$
\mathcal{V}_{*}=\mathcal{V}_{0}\left|\int_{0}^{t_{c}} d t e^{3 t} \int_{\sigma_{A}}^{\sigma_{\max }} d \sigma J_{\Phi}\right|,
$$

where $\mathcal{V}_{0}$ is the initial homogeneous volume, $t_{c}$ is a cutoff time that we use to regularize the volumes of the hypersurfaces, following the method employed in $[14,17,18]$. Therefore, substituting Eq. (20) in Eq. (18), we get

$$
\mathcal{V}_{*}=\mathcal{V}_{0}\left(\frac{4 C_{0}}{\kappa}\right) \frac{\Phi_{*}}{(2 \omega)^{1 / 2}}\left(\sigma_{\max }-\sigma_{A}\right) e^{8 \pi \omega \gamma_{1} \Phi_{*}} \frac{e^{\left(3-\gamma_{1}\right) t_{c}}}{3-\gamma_{1}}
$$

On the other hand, the volume $\mathcal{V}(\sigma, \Phi)$ of arbitrary hypersurfaces $(\sigma, \Phi)=$ const that are undergoing inflation is determined by the probability flux at $(\sigma, \Phi)$ across a line element located along the classical trajectory at that point. I.e., $|(\vec{J} \cdot \hat{l}) d t|$, where $\hat{l}$ is the tangent vector to Eq. (13). The expression is

$$
\mathcal{V}(\sigma, \Phi)=\mathcal{V}_{0}\left|\int_{0}^{t_{c}} d t e^{3 t}(\vec{J} \cdot \hat{l})\right|,
$$

and thus

$$
\begin{aligned}
\mathcal{V}(\sigma, \Phi)= & \mathcal{V}_{0} \frac{2 C_{0} \kappa}{\left(1+\omega^{2} \kappa^{2}\right)^{1 / 2}} \Phi\left[\kappa \omega \Phi^{1 / 2}+\frac{1}{\kappa}\left(\frac{2}{\omega}\right)^{1 / 2}\right] \\
& \times \exp \left(8 \pi \omega \gamma_{1} \Phi\right) \frac{e^{\left(3-\gamma_{1}\right) t_{c}}}{3-\gamma_{1}}
\end{aligned}
$$

Therefore the volume ratio $r$ of the hypersurface $(\sigma, \Phi)$ with respect to the thermalized regions is given by

$$
\begin{aligned}
r= & \frac{\mathcal{V}(\sigma, \Phi)}{\mathcal{V}_{*}} \propto \Phi\left[\kappa \omega \Phi^{1 / 2}+\frac{1}{\kappa}\left(\frac{2}{\omega}\right)^{1 / 2}\right] \\
& \times \exp \left[8 \pi \omega \gamma_{1}\left(\Phi-\Phi_{*}\right)\right] \sim \Phi^{3 / 2} \exp (24 \pi \omega \Phi),
\end{aligned}
$$

which is totally independent of $\sigma$, as are Eqs. (20), (24). We note the tendency towards larger values of $\Phi$, and indeed the likeliest value that is attained is $\Phi_{*}$. The ensembles of hypersurfaces $\Phi=$ const that are undergoing inflation are equally likely and occupy the same fraction of the total volume. These correspond to the horizontal cross-sections in the range $0<\Phi<\Phi_{*}$ in Fig. 2. In the limit of large $\omega$ this trend is preserved, except in the case when $\omega<0$. In that case, Eq. (25) yields the greatest likelihood for the smallest values of $\Phi$, i.e. $\Phi \approx \Phi_{0}$.

\section{SPECTRUM OF FLUCTUATIONS}

In order to calculate the resulting spectrum of fluctuations we need to derive the amplitudes of the typical quantum fluctuations in this model. In addition to the classical drift given by the slow-roll solutions, the stochastic nature of inflation exerts a quantum force over distances larger than $H^{-1}$ that can be described by [6]

$$
\begin{aligned}
& \dot{\sigma}=-\frac{1}{3 H} V^{\prime}(\sigma)+\frac{H^{3 / 2}}{2 \pi} \zeta(t) \\
& \dot{\Phi}=2 \frac{H}{\omega} \Phi+\frac{H^{3 / 2}}{2 \pi}\left(\frac{\Phi}{2 \omega}\right)^{3 / 4} \xi(t),
\end{aligned}
$$

where the Gaussian variables $\zeta, \xi$ satisfy $\left\langle\zeta\left(t_{1}\right) \zeta\left(t_{2}\right)\right\rangle$ $=\left\langle\xi\left(t_{1}\right) \xi\left(t_{2}\right)\right\rangle=\delta\left(t_{1}-t_{2}\right)$ and $\left\langle\zeta\left(t_{1}\right) \xi\left(t_{2}\right)\right\rangle=0$. Naturally Eq. (26) applies in the slow-roll regime, such that $\sigma_{A} \lesssim \sigma$ $\lesssim \sigma_{\max }$, and for arbitrarily large fields $\sigma \gg \sigma_{\max }$ the classical kinetic and potential energies are negligible, and $\sigma$ becomes stationary, whereas quantum fluctuations lead to changes in $\Phi$. The quantum jumps $(\delta \sigma, \delta \Phi)$ around an arbitrary hypersurface $(\sigma, \Phi)$ are distributed according to

$$
d P(\delta \sigma, \delta \Phi) \sim \frac{\mathcal{V}(\Phi+\delta \Phi)}{\mathcal{V}(\Phi)} d P_{0}(\delta \sigma, \delta \Phi)
$$

where $d P_{0}$ is a Gaussian distribution

$$
d P_{0}(\delta \sigma, \delta \Phi) \propto \exp \left[-\frac{(\delta \sigma)^{2}}{2 \Delta_{1}^{2}}-\frac{(\delta \Phi)^{2}}{2 \Delta_{2}^{2}}\right] d \delta \sigma d \delta \Phi,
$$

where $\Delta_{1} \equiv H / 2 \pi$ and $\Delta_{2} \equiv(\Phi / 2 \omega){ }^{1 / 2} H / 2 \pi$ are the variances of the fields and the preceding factor in the RHS of Eq. (28) is the volume ratio that determines the relative likelihood of the configuration $\Phi+\delta \Phi$ with respect to $\Phi$. From Eq. (25) we have

$$
\frac{\mathcal{V}(\Phi+\delta \Phi)}{\mathcal{V}(\Phi)} \approx\left(1+\frac{\delta \Phi}{\Phi}\right)^{3 / 2}\left(1+8 \pi \omega \gamma_{1} \delta \Phi\right)
$$

and therefore the typical quantum jumps, given by the stationary values of Eq. (28), are

$$
\begin{gathered}
\langle\delta \sigma\rangle \approx 0, \\
\langle\delta \Phi\rangle \approx\left(\gamma_{1} \Phi\right)^{1 / 2} \frac{H}{4 \pi},
\end{gathered}
$$


where the fluctuations in $\sigma$ remain Gaussian whereas the fluctuations of the BD field are not, due to the presence of the factor (30). As we have discussed in the previous section, in the case of $\omega>2 / 3$, the largest values of $\Phi$ are the likeliest ones, reaching a maximum at $\Phi \approx \Phi_{*}$. From Eq. (32) it follows that in this case the typical jumps are positive, and the evolution of the field towards $\Phi \approx \Phi_{*}$ is enhanced by quantum fluctuations of order $\sim \Phi^{1 / 2} H$.

In the case of $\omega<0$, we have seen from Eq. (25), that the smallest values of $\Phi$ are enhanced, i.e. $\Phi \approx \Phi_{0}$, and secondorder corrections to Eq. (32) contribute to suppress quantum jumps towards larger values of $\Phi$. The amplitude of these corrections is $\sim \omega V / \Phi^{2}$, and it is only significant for small values of $\sigma$ and $\kappa$ and large $\omega$. As one departs from $\Phi$ $\approx \Phi_{0}$ towards larger values of $\Phi$, the leading order of the fluctuations (32) rapidly dominates.

In the Einstein frame, the adiabatic density fluctuations with $\langle\delta \sigma\rangle \approx 0$ over distance scales of $H^{-1}$ are given by

$$
\frac{\delta \rho}{\rho}=-\frac{12}{5} H \omega \frac{\dot{\Phi}}{\Phi}\left(\dot{\sigma}^{2}+2 \omega \frac{\Phi^{2}}{\Phi}\right)^{-1} \delta \Phi,
$$

and therefore, by substituting Eq. (32) we get

$$
\left\langle\frac{\delta \rho}{\rho}\right\rangle \approx \frac{1}{10 \pi} \frac{H}{\Phi^{1 / 2}}
$$

which is to be evaluated for $N=65 e$-foldings after crossing EoI. The value of $\Phi$ in Eq. (34) is roughly $\Phi \approx \Phi_{*}$, as it will not change significantly after inflation, and $H$ is evaluated at $\sigma \approx \sigma_{\max }$. If the theory is correct, then the uniformity of the distribution (11) at EoI implies that $M_{\mathrm{p}}^{*} \sim 10^{19} \mathrm{GeV}$ throughout, as in our region of the universe, and $\kappa \sim 10^{-18} \mathrm{GeV}^{-1}$, and from Eq. (15) we have that $\sigma_{\max } \sim 10^{21} \mathrm{GeV}$. From the astrophysical constraint $\langle\delta \rho / \rho\rangle \lesssim 10^{-4}$, these estimates in turn imply that $V_{0} \lesssim 10^{58} e^{6 \omega} \mathrm{GeV}^{4}$, which in practical terms leaves $V_{0}$ unconstrained for conservative values of $\omega$ $\gtrsim 500$.

\section{CONCLUSIONS}

In this paper we have examined extended inflation with an exponential potential. The remarkable feature of this model is the prediction of a constant distribution of the Planck mass at the end of inflation, given by Eq. (11). The parameter $\kappa$ of the theory is therefore estimated via the observed Planck mass in this region of the universe, which in turn fixes the parameter $\sigma_{\max }$ that determines the range of values of $\sigma$ for which inflation takes place.

The amplitude of the potential $V_{0}$ is left unconstrained by astrophysical bounds on the spectrum of fluctuations, as described by the argument given in Sec. 4. The dynamics as is given in Sec. 2 and the likelihood distributions in Sec. 3 are shown to be insensitive to the numerical value of this parameter.

As is shown in Fig. 2, the BoI and EoI curves in this model cross at $\sigma=\sigma_{A}$ and the area enclosed between them is thus infinite. However the breakdown of the slow-roll approximation for the exponential potential over the range $\sigma$ $\gtrsim \sigma_{\max }$ [where $\sigma_{\max }$ is given by Eq. (15)] implies that in practical terms only a finite region of the $(\sigma, \Phi)$ plane undergoes inflation. In the classification of [16] this means that the exponential potential is class $I$, i.e. the values of the fields at EoI remain finite.

\section{ACKNOWLEDGMENTS}

A.M. is supported by the Inlaks foundation and the ORS. We thank Kei-ichi Maeda, Andrew Liddle, John Barrow, Dominik Schwarz, and Reza Tavakol for useful discussions, and A.M. acknowledges use of the Starlink computer system at the University of Sussex.
[1] P. A. M. Dirac, Nature (London) 139, 323 (1937); Proc. R. Soc. London A165, 199 (1938).

[2] D. W. Sciama, Mon. Not. R. Astron. Soc. 113, 34 (1953).

[3] C. Brans and R. H. Dicke, Phys. Rev. 124, 925 (1961); R. H. Dicke, ibid. 125, 2163 (1961); C. Brans, ibid. 125, 2194 (1961).

[4] J. D. Barrow, Mon. Not. R. Astron. Soc. 282, 1397 (1996); J. D. Barrow and P. Parsons, Phys. Rev. D 54, 1906 (1997); A. R. Liddle, A. Mazumdar, and J. D. Barrow, ibid. 58, 027302 (1998).

[5] A. D. Linde, Particle Physics and Inflationary Cosmology (Harwood, Academic, Chur, Switzerland 1990); A. S. Goncharov, A. D. Linde, and V. F. Mukhanov, Int. J. Mod. Phys. A 2, 561 (1987).

[6] A. A. Starobinsky, in Current Topics in Field Theory, Quantum Gravity and Strings, edited H. J. de Vega and N. Sánchez, Lecture Notes in Physics 206 (Springer, Heidelberg 1986); M.
Mijić, Phys. Rev. D 42, 2469 (1990).

[7] N. G. Turok and S. W. Hawking, gr-qc/9803156.

[8] D. La and P. J. Steinhardt, Phys. Rev. Lett. 62, 376 (1989); D. La, P. J. Steinhardt, and E. Bertschinger, Phys. Lett. B 231, 231 (1989); J. D. Barrow and K. Maeda, Nucl. Phys. B341, 294 (1990)

[9] D. S. Goldwirth and H. W. Zaglauer, Phys. Rev. Lett. 67, 3639 (1991); T. Damour and K. Nordtvedt, Phys. Rev. D 48, 3436 (1993); J. D. Barrow, ibid. 51, 2729 (1995); T. Chiba, Class. Quantum Grav. 14, 2951 (1997).

[10] J. Garcia-Bellido, A. D. Linde, and D. A. Linde, Phys. Rev. D 50, 730 (1994).

[11] E. Weinberg, Phys. Rev. D 40, 3950 (1989).

[12] A. A. Starobinsky and J. Yokoyama, in Proceedings of the 4th Workshop on General Relativity and Gravitation, Kyoto, Japan, 1994, astro-ph/9502002.

[13] M. P. Susperregi, Phys. Rev. D 55, 560 (1997). 
[14] M. P. Susperregi, gr-qc/9712031.

[15] T. Damour and V. F. Mukhanov, Phys. Rev. Lett. 80, 3440 (1998).

[16] M. P. Susperregi (in preparation).
[17] A. Vilenkin, Phys. Rev. D 52, 3365 (1995); S. Winitzki and A. Vilenkin, ibid. 53, 4298 (1996).

[18] A. D. Linde and A. Mezhlumian, Phys. Rev. D 53, 4267 (1996); J. Garriga and A. Vilenkin, ibid. 57, 2230 (1998). 